\title{
FILÓSOFOS Y FILOSOFÍAS
}



Fecha de entrega: 21 de octubre de 2008

Fecha de aprobación: 18 de marzo de 2009

\section{EL VIAJERO EXPLORADOR Y LAS SELVAS SIN CAMINOS DEL NUEVO MUNDO}

\section{Acercamientos al método fenomenológico de Husserl ${ }^{1}$}

\section{THE TRAVELLER EXPLORER AND THE FORESTS WITHOUT ROADS OF THE NEW WORLD AN APPROACH TO THE PHENOMENOLOGICAL METHOD OF HUSSERL}

Omar Esteban Barbosa Martínez $z^{2}$

\section{Resumen}

En este escrito se aborda el problema de la fenomenología como filosofía y como método. Asimismo, se destacan los elementos metodológicos que Husserl ofrece en sus obras. Este proceso metodológico es posible encontrarlo en la forma de exponer el ejercicio propio del filosofar: como geógrafo. Para ello tomaremos como referencia el método fenomenológico expuesto por el propio Husserl. En primer término, esbozaremos los fundamentos de la fenomenología husserliana, luego estudiaremos los elementos que conforman el método fenomenológico.

1 Este artículo es resultado de la pasantía doctoral que se realizó en la Universidad de Texas en Arlington, bajo la dirección del doctor Harry P. Reeder. Agradezco al doctor Reeder sus correcciones y comentarios. La pasantía se realiza gracias a la financiación que brinda la Universidad Santo Tomás (Bogotá, Colombia), para la realización de estudios de Doctorado.

2 Estudiante del Doctorado Interinstitucional en Educación de la Universidad Pedagógica Nacional, en el Énfasis Filosofía y Enseñanza de la Filosofía. Director de tesis: doctor Harry P. Reeder. 


\title{
Palabras clave
}

Fenomenología, método fenomenológico, conciencia, reducción progresiva.

\begin{abstract}
This essay approaches the problem of phenomenology as philosophy and as method, and the methodological elements that stand out in Husserl's works. The methodological process may be found in Husserl's analogy for doing phenomenology: as geographer. First we will sketch the foundations of the phenomenology of Husserl, and then we will study the elements that make up the phenomenological method.
\end{abstract}

\section{Key words}

Phenomenology, phenomenological method, consciousness, progressive reduction.

La principal tarea... será buscar caminos por los cuales quepa superar a trozos... las más que grandes dificultades de la entrada en este nuevo mundo.

Husserl, Edmund, Ideas I

\section{Introducción}

Nuestro principal interés es hacer un acercamiento al método fenomenológico de Husserl. Aunque inicialmente Husserl quizo proponer una filosofía fundamentado en el psicologismo, encontró que las leyes lógicas no pueden tener un fundamento psicológico, sino que dichos actos lógicos son actos de naturaleza intencional que tienen sus correlatos en la conciencia como conciencia intencional.

Así es como, posteriormente, Husserl va a dedicar sus reflexiones centrado en esta conciencia intencional. Estas reflexiones en torno a la conciencia lo llevan a describir las estructuras fundantes de dicha conciencia. Para ello se vale de un proceso metódico que le permite no sólo describir la conciencia, sino también la necesidad de plantear los componentes metódicos de dicho proceso. Siguiendo la analogía propuesta por el mismo Husserl de ser un geógrafo en dicha descripción, haremos un viaje por estas tierras nuevas, describiendo la concepción husserliana de la conciencia e indicando los elementos del método fenomenológico. Veamos en primer lugar este punto de partida.

\section{El viajero explorador}

Husserl en el $\S 5$ del Epílogo y en el $§ 96$ de Ideas I, (Husserl, 1997, pp. 235 y 388) se considera a sí mismo como un "geógrafo", un geógrafo viajero, un explorador:

[...] quien durante decenios no especula sobre una nueva Atlántida, sino que se ha metido realmente por las selvas sin caminos de un nuevo continente y ha hecho los primeros esfuerzos para cultivarlo, no se dejará extraviar por negativa alguna de los geógrafos que juzgan de las noticias por sus propios hábitos empíricos y mentales -pero que también se ahorran el esfuerzo de 
hacer un viaje a las nuevas tierras (Husserl, 1997, Epílogo § 5, p. 388).

Así:

[...] nuestro proceder es el de un viajero que explora una parte desconocida del mundo, describiendo cuidadosamente lo que se le presenta a lo largo de sus caminos no trillados y que no siempre serán los más cortos. A tal viajero puede henchirle la segura conciencia de dar expresión a lo que tenía que expresarse de acuerdo con el momento y las circunstancias, lo cual, por ser fiel reproducción de lo visto, conservará por siempre su valor, aunque nuevas exploraciones requerirán nuevas descripciones con múltiples correcciones. Con semejante espíritu queremos ser en adelante un fiel expositor de los hechos fenomenológicos, conservando por lo demás el habitus de una íntima libertad incluso frente a nuestras propias descripciones (Husserl, 1997, p. 235).

Siguiendo esta comparación, trataremos de hacer aquí una descripción geográfica de la fenomenología y su método, propuestos por Husserl y sus más cercanos seguidores, de aquellos que de alguna manera contribuyeron a su edificación. Para ello, esbozando los fundamentos de la fenomenología husserliana y desde allí indicar los elementos que conforman el método fenomenológico.

\section{La entrada en este nuevo mundo}

¿Cómo, entonces, explorar esa parte desconocida del mundo y ver aquellos paisajes, en donde montañas, honduras, profundidades, ríos y horizontes, se dibujan en su obra escrita? Para lograr nuestro propósito es necesario que veamos algunos antecedentes que sirvieron de base para su propuesta fenomenológica. Para ello intentaremos recrear esa bitácora de aquellos viajes y hechos fenomenológicos que pudo ver en aquellos caminos no trillados, ver aquella señalada tierra prometida por Husserl y levantar la cartografía de su propuesta fenomenológica. Es así como "la principal tarea... será buscar caminos por los cuales quepa superar a trozos... las más que grandes dificultades de la entrada en este nuevo mundo" (Husserl, 1997, Introducción, p. 9).

\section{La posibilidad del nuevo mundo}

Aunque inicialmente sus preocupaciones se centran en las matemáticas, Husserl tiene noticias de un posible nuevo mundo a través de otro visionario, Brentano, quien influyó con su idea de la intencionalidad, y de Carl Stumpf de quien toma la idea de estado de cosas (Sacheverhalt), emprendiendo así su camino por los campos del vasto mundo de la filosofía. Decisión que le lleva a realizar sus primeros viajes, combinando las matemáticas, la psicología y la filosofía, tomando como plan orientador a la psicología descriptiva en sus dos primeras obras, Über den Begriff der Zahl (Sobre el Concepto de Número, 1887), y Philosophie der Arithmetik (Filosofía de la Aritmética, 1891). Pero estas primeras incursiones son simplemente la preparación para su "gran viaje" a esas tierras aun sin explorar.

Es así como Husserl inicia su viaje por las selvas sin caminos de un nuevo continente en el territorio de las matemáticas, tratando de aclararse a sí mismo el sentido de la experiencia humana. Teniendo como brújula los marcos teóricos del psicologismo, analiza los conceptos fundamentales de las matemáticas. Unidad, multiplicidad, cantidad, etc., conceptos que aborda en Filosofía de la Aritmética. Pero como buen expedicionario su interés se 
dirige sobre un territorio aún no explorado por los psicologistas, su mirada se fija en aquello que caracteriza la actividad del matemático. Guiado por lo expresado por Brentano, para quien la intencionalidad, es decir, su referencia esencial a algo, es la característica fundamental que distingue un hecho psíquico de un hecho natural, plantea que el número es el fruto de la actividad del matemático. Esta actividad, según Husserl, no puede ser expresada en términos causales o debido a condiciones naturales, sino que su origen subyace en la aprehensión de la intencionalidad propia de dicha actividad. Por tanto, la esencia del acto psíquico se da de manifiesto en la capacidad de dar sentido y de poder orientarse intencionalmente en este sentido.

Así durante nueve años y bajo la influencia de Bolzano, Leibniz y Lotze dirige su atención hacia la teoría platónica del conocimiento y en un ambiente en el que se disputa en torno a la naturaleza y fundamento de la lógica, permiten a Husserl redireccionar su plan y pensamiento hacia un anti-psicologismo, tendencia que se ve reflejada en su obra Logische Untersuchungen (Investigaciones Lógicas, 1900-1901).

Lo que Husserl descubre no es el mundoen-sí, ya que es ese mundo que ha estado ahí, es el mundo-ahí, que no cambia, sino que es aquello que le sucede al viajero, sus estructuras internas de conciencia que le permiten aprehender ese mundo de una o de otra manera, es más, descubre que el mundo es mundo por lo que le sucede al viajero. Es desde aquí que decide hacer ese viaje al interior de las estructuras de la conciencia en un nuevo giro copernicano.

\section{En los pantanos de inestable oscuridad}

La aplicación de los nuevos conceptos que fueron primeramente incorporados en su cartografía del nuevo mundo, le permiten publicar Ideen zu einer reinen Phänomenologie und phänomenologischen Philosophie (Ideas relativas a una fenomenología pura y una filosofía fenomenológica) en 1913, y en la reorientación, en una segunda edición, del plan trazado inicialmente para las Logische Untersuchungen (1913).

Reconoce que internarse en la conciencia era internarse en un terreno pantanoso carente de fundamento, por eso:

[...] me decidí a renunciar a alcanzar grandes metas en el trabajo filosófico; y ser feliz si podía tan sólo alcanzar con mi trabajo, aquí o allá, el más pequeño fundamento, en los pantanos de inestable oscuridad. Los más pequeños fundamentos sobre los cuales yo realmente pudiera ponerme en pie, justamente en la evidencia del firme estar en pie (Carta de Husserl a Cairns Husserl, 1930, p. 27).

Consideramos Ideas I como la puerta de entrada a las nuevas tierras, a un nuevo mundo, ya que es allí donde su manera de ver va a cambiar radicalmente y empieza a trazar los primeros mapas que van a guiar su viaje por estas selvas sin caminos de un nuevo continente, el continente de:

[...]la fenomenología pura, a la que queremos abrirnos el acceso en lo que sigue -la misma que en las Investigaciones lógicas se abrió campo por primera vez y cuyo sentido se me hizo cada vez más hondo y rico en el trabajo continuo del último decenio-" (Husserl, 1997, Introducción, p. 8).

Sin embargo, estos primeros intentos por cartografiar la "estructura del estado consciente" le llevan a Husserl a nuevos descubrimientos, 
claves para sus exploraciones posteriores. Entre ellos están el distinguir entre el acto de la conciencia (nóesis) y los fenómenos a los que se dirige y que son objetos de consciencia (noema, noémata). A esto hay que agregar que para llegar al conocimiento de las esencias (Wesen), la única vía de acceso posible sería eliminando todas las suposiciones o pre-juicios que se tengan sobre la existencia del mundo exterior. A este fundar lo llamó el método de la epojé y reducción fenomenológica, es decir: parentetización, poner entre paréntesis lo que se supone como ya sabido, para así intentar llegar a las esencias o eidos de las cosas.

Tenemos, así con la epojé fenomenológica una de las primeras regiones alcanzadas dentro del plan de constitución de la conciencia. "Nuestra meta, que podemos formular también como la conquista de una nueva región del ser, hasta aquí no deslindada en lo que tiene de propio, y que, como toda auténtica región, es una región de ser individual' (Husserl, 1997, p. 75).

\section{El camino de la conquista}

En 1935, haciendo una retrospectiva de su trabajo, reconoce que en su intento por establecer esta aprehensión de la intencionalidad, encuentra que existe una correlación de los elementos subjetivos y objetivos, pero no es sino hasta 1898 cuando toma plena conciencia de esta correlación, al darse cuenta que en esta actividad también se encuentra de manera implícita todo un sistema de vivencias. Dicho sistema, al parecer, se da de forma apriorística y desde entonces, "todo el trabajo de mi vida estuvo dominado por la tarea de elaborar sistemáticamente este a priori de la correlación” (Husserl, 1991, p. 169).

No es sino hasta 1901, con la publicación Investigaciones Lógicas, cuando Husserl inicia la exploración y construcción de ma- nera sistemática de esta nueva zona entre la subjetividad y la objetividad: la correlación. Para ello, en esta obra Husserl quiere "ir más allá" del campo de la experiencia del matemático, adentrándose en los aun no explorados extensos campos de la experiencia humana.

Ya no se pregunta por la ciencia matemática. Ahora su mirada se dirige hacia la ciencia en cuanto ciencia, la ciencia en sí misma, hacia su fundamento. Pero para responder a esta pregunta es necesario elaborar una herramienta "limpia" de cualquier pre-juicio, es decir, concebir una "lógica pura", como ciencia teórica, autónoma, a priori. Así es como en el primer volumen de las Investigaciones Husserl se dedica a refutar el psicologismo, que consideraba las leyes lógicas como leyes de hechos, de hechos psíquicos, de costumbres de pensar, de hábitos de economía mental. Por tanto, la primera tarea es asegurar la objetividad de las formas y leyes lógicas contra toda interpretación subjetiva.

Descubierta esta objetividad de las leyes y formas lógicas, Husserl emprende el camino de conquistar, en el segundo volumen de Investigaciones, aquellas "verdades en si", es decir, aquellos fundamentos lógicos de la ciencia. Husserl incursiona en el vasto campo de lo que aparece a la conciencia intencional. Para ello describe, tanto el acto de pensamiento como su correspondiente objeto. Este objeto es el fenómeno en cuanto tal. Por tanto, el fenómeno es la conciencia de la cosa en cuanto pensada.

Husserl va a llamar a este dirigir, la "mirada" o atención hacia los actos como la "reducción". Es aquí, en Investigaciones Lógicas, en donde prueba el proceso que se debe seguir. En este proceso de significación se aplican tres momentos íntimamente ligados uno del otro: la tematización, la variación, la ideación. El primer paso es dar forma a algo que en apariencia es imposible considerarlo como un "objeto" en sí mismo. A este primer 
paso, Husserl lo llama tematización. Para lograrlo es necesario proceder paso a paso en su exploración, hasta convertirlo en objeto de un acto de conciencia. Alcanzado este primer paso se procede a la variación, es decir, a aquellas modificaciones que puedo hacer al objeto en sus relaciones. Este ejercicio tiene como finalidad poner de manifiesto aquellos elementos que no pueden ser eliminados sin eliminar el todo anteriormente tematizado. Por último, teniendo lo que permanece invariable en este ejercicio de variación, nos encontramos con la ideación, es decir, con aquello que conforma la esencia o sentido eidético del objeto. Y es hacia ese sentido que se dirige la mirada, por ser los elementos que constituyen una unidad del objeto de un acto de conciencia.

En síntesis, podemos decir que el camino de significación hasta aquí conquistado, es la explicitación del proceso mismo de lo que sucede en la "intuición de una esencia". Posteriormente, en la Idea de la Fenomenología, considera que los terrenos por él vislumbrados y explorados obedecen a esa nueva manera de ver el mundo, es por eso que considera que "la fenomenología es la doctrina universal de las esencias, en la que halla su lugar la ciencia de la esencia del conocimiento" (Husserl, 1982, p. 92).

\section{Desde la superficie se ve uno conducido hacia las profundidades}

Por referencias de un "visionario" tuvo noticias de otras formas de entender el mundo, la intuición y su intencionalidad, y quiso incursionar por esos nuevos "horizontes". Pero encuentra que "desde la superficie se ve uno conducido hacia las profundidades" (Husserl, 1991, p. 366). A partir de ese momento las "cosas" serían diferentes, todo sería visto con "ojos diferentes", El "mundo" no volvería a ser "lo mismo" en esas fecundas honduras de la experiencia. Así todo el trabajo de su vida estará guiado por esa intuición primigenia y originaria "todo el trabajo de mi vida estuvo dominado por la tarea de elaborar sistemáticamente este a priori de la correlación" (Husserl, 1991, pp. 169-170).

Una cosa es ver, esto es, en general, vivenciar, experimentar, tener en el campo de percepción, y otra ejecutar el ver en el sentido especial, percatándose, "vivir" en el ver de una manera eminente, actuar en cuanto yo en el sentido especial "creyendo", juzgando, ejecutar un acto de juzgar como un cogito, estar dirigido a lo objetivo con mirada activa, el estardirigido específicamente mentante (Husserl, 1997, p. 33).

Pensemos un poco en la profundidad. Estoy en la playa y veo su cristalina agua que golpea mis pies. Esa agua cristalina, a pesar de estar ahí en un constante y hasta aparente vaivén caótico, me permite ver su fondo, pero doy unos cuantos pasos mar adentro y las cosas ya no se ven tan claras como al comienzo. Son más oscuras y es más difícil ver su fondo. Llegar hasta ese fondo implica meterme en sus profundidades. Pero no lo veré por la oscuridad allí reinante. Al reflexionar sobre la profundidad, encuentra que:

[...] sólo cuando en la conciencia contemporánea se haya impuesto la separación definitiva entre las dos clases de filosofía [filosofía de la cosmovisión y filosofía científica], cabrá pensar en dar a la filosofía la forma y el lenguaje de la ciencia genuina y hacerle reconocer como imperfección lo que tantas veces se ha admirado y hasta imitado en ella: a saber, su obscuridad profunda. La profundidad es un síntoma del caos que la verdadera ciencia debe ordenar en cosmos, en un orden simple, 
completamente claro y resuelto. La verdadera ciencia, en todo el alcance de su doctrina real, ignora la profundidad. Todo fragmento de ciencia acabada es un todo compuesto de elementos del pensamiento, cada uno de los cuales es comprendido de inmediato, o sea no es profundo. La profundidad pertenece a la sabiduría; la distinción conceptual y la claridad pertenecen a la teoría estricta. Traducir las conjeturas de la profundidad en formas racionales inequívocas constituye el proceso esencial de la reconstitución de las ciencias estrictas. También las ciencias exactas han tenido largos períodos de profundidad y del mismo modo que las ciencias en las luchas del Renacimiento, la filosofía pasará ---así lo espero--- en las luchas del presente, desde el grado de la profundidad al de la claridad científica (Husserl, sf., p. 70).

\section{Lo aventurado en su fundación}

Las noticias publicadas en Investigaciones Lógicas, atrajeron un sin número de colonizadores, que guiados por lo logrado por el pionero Husserl, se entregaron de lleno a la descripción de las esencias de cada una de las regiones del ser. Aunque muchos no alcanzaron a vislumbrar lo aventurado por su fundador: aquella conciencia intencional que supone la trascendencia de las cosas y de las esencias.

Sin embargo, esto no es un obstáculo para un pionero. El terreno conquistado por Husserl en Investigaciones Lógicas, le permitió exponer, en contra del pensamiento tradicional, el carácter intencional de la conciencia, pero sólo en una dirección, en el contenido objetivo de la experiencia humana. Ahora dirige su mirada hacia el campo del sujeto mismo de la correlación y, más específicamente, hacia la estructura interna de la conciencia misma del explorador. En esta esfera de la experiencia humana encuentra una gran riqueza. Riqueza que es expuesta en 1913 con la publicación de su libro Ideas relativas a una fenomenología pura y una filosofía fenomenológica (Ideas I).

Tratemos de seguir esa veta abierta por Husserl en la que pone de manifiesto la conciencia misma. Para ello Husserl nos invita a reflexionar sobre el modo de estar, de vivir en el mundo.

Dicho modo de estar es comparable a estar dentro de una cueva, un estar perdido en el mundo. El hombre, en su vida cotidiana, se experimenta como un ser más entre los seres, es decir, está en el mundo con las cosas que le rodean, así como con los demás hombres. Implícitamente "sabe" que "ese mundo" está ahí, y que él forma parte de ese mundo, siempre en constante relación con ese mundo, bien sea extraviado, interpelado, perturbado, etc. Vive dentro de ese mundo, forma parte de él, pero, también reconoce que ese mundo transcurre sin él. Este modo de vivir constituye nuestra actitud natural, y a la forma de poner la existencia de las cosas frente a nosotros, Husserl la llama tesis general del mundo.

Es así como Husserl considera que por estar el hombre dentro de ese mundo, no podemos ver ese mundo. Es un ver sin poder ver. Por eso nos propone Husserl cambiar la mirada, ver el mundo de manera distinta, de otra manera, dirigir nuestra atención hacia aquello que vemos con una mirada diferente y para ello nos propone salir de ese mundo volviendo la mirada hacia ese mundo y tomando distancia sobre ese mundo, pero sin alejarnos de él, es decir, cambiar nuestra actitud. A esta nueva actitud Husserl la denomina actitud reflexiva.

Encontrada la veta, queda ahora seguirla. "Desde la superficie se ve uno conducido hacia las profundidades". Para ello Husserl 
propone seguir un camino que conduce hacia la conciencia pura. Por ser aquello en el cual "queda algo", Husserl lo denominó con el término de reducción.

En Ideas I, esta operación con que se la conquista implica diversos momentos.

Metódicamente se descompondrá esta operación en diversos pasos que "desconectarán” o "colocarán entre paréntesis", con lo que nuestro método tomará el carácter de una reducción progresiva. Por ello hablaremos, e incluso preponderantemente, de reducciones fenomenológicas (aunque también, y en vista de su unidad total, de la reducción fenomenológica en singular), o, bajo el punto de vista epistemológico, también de reducciones trascendentales (Husserl, 1997, p. 77).

Para nuestro ejercicio de visualización panorámica de la fenomenología, en el que marcamos los principales puntos de referencia, indicaremos los principales:

Un primer momento de esta operación es lo que Husserl llama épojé. Se trata de poner "entre paréntesis" aquello que es propio de la actitud natural, es decir, liberarnos de su objetivismo para suprimir la presión de la realidad y tomar distancia frente a ella y así descubrir al sujeto de la correlación hombremundo. En otras palabras, esta función metódica suspende la tesis general del mundo, desconectando y evitando cualquier juicio que podamos hacer sobre ese mundo. Su finalidad no se dirige, por tanto, a la existencia y al conocimiento del mundo natural, ni a los fundamentos de la certeza del Cogito, sino a dirigir la mirada hacia la conciencia y hacia todo lo que en ella se da.

Suspendida la tesis general del mundo, puesto entre paréntesis, queda un residuo: la conciencia de algo. Al quedar este residuo de la "conciencia de algo", puedo entonces detenerme a describir el sentido o esencia de ese algo. Y para ello utilizó el proceso de tematización, variación e ideación propuesto en Investigaciones Lógicas, no para demostrar la certeza de ese algo sino su sentido. Esta segunda operación, Husserl la llama reducción eidética.

Pero si queremos profundizar en esta veta, es necesario ir más allá de los resultados alcanzados en la reducción eidética. Ya que se ha alcanzado el sentido como objeto de la conciencia intencional, es decir, como correlato intencional de un acto, es posible dirigir ahora la mirada hacia la conciencia en sí misma, es decir, hacia la correlación intencional en sí misma. Esta operación, que Husserl llama reducción trascendental, trata de descubrir la conciencia misma en su movimiento, en su dinámica, en su vida, en su donación de sentido.

Por tanto, podemos decir que la operación metodológica de reducción progresiva aplicada a aquella veta de la experiencia humana, pone de manifiesto capas más profundas de la conciencia y de sus vivencias. En la primera pone al descubierto la intencionalidad psicológica con sus objetos; en la segunda, la intencionalidad trascendental que piensa el mundo y el sentido del mundo y, en la tercera, la intencionalidad como origen del sentido del mundo.

Hecho este recorrido por la conciencia y sus correlaciones, según lo visto por Husserl, queda ahora hacer nuevas exploraciones. Y estas exploraciones requerirán nuevas descripciones con múltiples correcciones, que se irán complementando a lo ya planteado por Husserl y sus seguidores. Esta es la invitación que nos extiende Husserl. Indicación que debemos tener en cuenta en nuestras aplicaciones del método en las distintas investigaciones que se llevan a cabo. 


\section{Referencias}

Buckley, R. En Edmund Husserl, McGill University (Tomado de Encyclopedia of Phenomenology, Lester Embree (ed.).. Contributions to Phenomenology. Dordrecht/Boston/London: Kluwer Academic Publishers, 1997, pp. 326-331.) Consultado el 05 de febrero de 2009 de http://www.filosoficas.unam.mx/ zirion/ Husserl-Buckley.pdf

Husserl, E. (1930). Carta de Edmund Husserl a Dorian Cairos. En Praxis Filosófica, 10-11. Universidad del Valle. (Julio César Vargas B., trad.). Departamento de Filosofía, Universidad del Valle.

Husserl, E. (1982). Investigaciones lógicas I y II. Madrid: Alianza Editorial.

Husserl, E. (1982). La idea de la fenomenología. México, Editorial: Fondo de Cultura Económica. Versión digital en: http:// www.scribd.com/doc/7177267/HusserlE-La-Idea-de-La-Fenomenologia

Husserl, E. (1982). La idea de la fenomenología. Cinco lecciones. (Miguel GarcíaBaró, trad. y presentación). MéxicoMadrid-Buenos Aires: Fondo de Cultura Económica; Ediciones FCE España.

Husserl, E. (1991). La crisis de las ciencias europeas y la fenomenología trascendental. (Salvador Más y Jacobo Muñoz, y trads. y nota editorial). Barcelona: Crítica.
Husserl, E. (1997). Ideas relativas a una fenomenología pura y una filosofía fenomenológica. Libro Primero: Introducción general a la fenomenología pura. (J. Gaos, trad.). México: Fondo de Cultura Económica.

Husserl, E. (1997). Ideas relativas a una fenomenología pura y una filosofía fenomenológica. Libro Segundo: investigaciones fenomenológicas sobre la constitución. México: Fondo de Cultura Económica.

Husserl, E. (s.f) La filosofía como ciencia estricta. (Elsa Tabernig, trad.). Buenos Aires: Editorial Nova. Versión digital en: http://www.scribd.com/doc/9260097/ Edmund-Husserl-La-Filosofia-ComoCiencia-Estricta.

Husserl, E. (1986). Meditaciones cartesianas. (José Gaos, trad. primeras cuatro Meditaciones). (Miguel García-Baró, trad. Meditación Quinta). México: Fondo de Cultura Económica.

Zirión Quijano, A. (s.f). Diccionario Husserl. Léxico bilingüe (alemán y español) de expresiones definidas a partir de las obras de Edmund Husserl (1859-1938). Instituto de Investigaciones Filosóficas de la Universidad Nacional Autónoma de México. En: http://www.clafen.org/ diccionariohusserl/es/?action=siteaccess 